\section{DOI: 10.21315/aos2019.14.2.404}

ARTICLE INFO

Submitted: 09/08/2019

Accepted: 15/12/2019

Online: 30/12/2019

\section{Treatment of Cancer: Need for Paradigm Shift}

\author{
Thirumulu Ponnuraj Kannan ${ }^{a, b}$ \\ ${ }^{a}$ School of Dental Sciences, Universiti Sains Malaysia, 16150 Kubang \\ Kerian, Kelantan, Malaysia \\ ${ }^{b}$ Human Genome Centre, School of Medical Sciences, Universiti Sains \\ Malaysia, 16150 Kubang Kerian, Kelantan, Malaysia
}

E-mail: kannan@usm.my

To cite this article: Kannan TP (2019). Treatment of cancer: need for paradigm shift. Arch Orofac Sci, 14(2): 75-76. https://doi.org/10.21315/aos2019.14.2.404

To link to this article: https://doi.org/10.21315/aos2019.14.2.404

The word 'cancer' obviously provokes a sense of immediate trepidation and has unwittingly 'metastasised' the human perception solely eliciting a sickening feeling of hopelessness despite the unabated claims on improved treatments. Such current treatment modalities include surgery, chemotherapy, radiation therapy and traditional, herbal and home therapies. An in-depth discussion on the advantages and disadvantages of these treatment modalities is beyond the horizon of this essay. The objective is to highlight the treatment cost as a cardinal factor impacting the emotional state of the patients as well as the caretakers especially in view of the equivocal nature of 'cure' in many a cancer.

Radiation therapy, a fairly cost-effective method is beneficial in destroying the cells in and around the target area. Cryosurgery, an improved surgical modality leaves questions on long term effectiveness unanswered. Many of the other techniques such as biological therapy and gene therapy are still in the research level and if were to be implemented would involve huge expenditure. Prompted by the proven efficacy of vaccines towards controlling diseases like smallpox and polio, cancer researchers have also shown the possibility of vaccine related control of certain selecting types of cancers. However, generating vaccines for a majority of other types of cancers is still in its infancy.

World Health Organization (WHO) has projected a catastrophic increase in the death due to cancer from 9.6 million in 2018 to 13 million in 2030. This apart, the estimated increase in the number of new cancer cases between 2008 and 2030 would surpass $80 \%$ in low-income countries compared to $40 \%$ expected in high-income countries (WHO, 2019). Excepting the limited types of cancers that have been shown as curable, the scope for containing many of the other categories of cancers appears bleak despite decades of research. Cancer is now understood to be largely caused by environmental factors, a more ominous revelation since such factors involved appear to be multiple and uncontainable. Had the cause been single and determinable, a cure could have been possible as was the case with smallpox. Research is indeed a necessity for solving problems in science; but one is impelled to wonder if such research ventures would prove a wild goose chase when increasing evidence indicate the cause to be environmental and multiple. Indeed, about $90 \%$ to $95 \%$ of cancers are attributed to possible environment and lifestyle factors (Anand et al., 2004) that are beyond human command to streamline. 
It is explicit that carcinogens exist as integral part in the state of nature and seeking to protect oneself from being exposed is an impossibility. It is a disturbing fact that the incidence of cancer is increasing among children and youngsters who apparently are still in the phase of leading a 'good lifestyle'. This social reality raises a pesky question whether to continue fanciful researches towards treatment and transfer their costs to the medication and treatment further burdening the patients or to explore a paradigm shift in facing the current cancer threat by considering it a global environmental problem to be treated on a subsidised basis as a social welfare measure.

Indeed, the major factor that worries a cancer patient next only to the disease itself is the cost involved in the treatment. It is a fact that none of the treatment regimens accepted as of proven effect is affordable by average income group in view of the exorbitant cost involved. Inevitably, except those who belong to the affordable class or who have comfortable insurance coverage, all the others find themselves either in the precarious condition of draining their limited resources or resigning themselves altogether from undergoing the treatment. This social reality impeding cure for an inestimable population needs to be taken cognizance of by the specialists involved in cancer cure as well as the concerned world welfare bodies. While the cost of treatment is exorbitant, there has grown another industry selling expensive herbal brand formulae and other food supplements professing to contain or cure cancer. Alongside, is an unholy trend that elevated cost of treatment is somewhat an assurance correlating with better alleviation of pain.

From the point of view of society's welfare, the real state of cancer appears to remind us of the prevalence of such epidemics as smallpox and polio in the past. It has to be recalled that thanks to the special eradication programmes launched by the WHO in 1967 for smallpox and the Global Polio Eradication Initiative in 1988 for polio, it was possible to completely eradicate these diseases. If only the treatment of smallpox or polio had been as costly as the treatment of cancer, a major part of human race would have vanished decades ago. It was the wellness of the society that constrained the cost of such items like polio vaccine, ivermectin etc. The opposite is the scenario in cancer treatment and drugs. The cost of cancer drugs has been shown to have doubled within the past decade - another proof that remedy via drugs is beyond the reach of the poor.

The scientific community cannot afford to be too optimistic towards advancing the treatments for cancer. One cannot ignore the plight of the patients on account of the cost of treating cancer. Cancer, a noncommunicable disease, is seen registering an increase while diseases that are infectious are showing a declining trend. Should we permit the current trend and let the treatment grow dearer or should we draw the attention of bodies like WHO to fund the treatment of cancer the same way smallpox, polio, malaria treatments were funded. It seeks to support the latter proposition.

\section{REFERENCES}

Anand P, Kunnumakkara AB, Sundaram C, Harikumar KB, Tharakan ST, Lai OS et al. (2004). Cancer is a preventable disease that requires major lifestyle changes. Pharm Res, 25(9): 2097-2116. https://doi.org/10.1007/ s1 1095-008-9661-9

World Health Organization (2019). Cancer. Key statistics. Retrieved 30 July 2019, from https://www.who.int/cancer/resources/ keyfacts/en/ 